\title{
Characteristics of Recipients of Red Blood Cell Concentrates in a German Federal State
}

\author{
Linda Schönborn $^{\mathrm{a}}$ Kerstin Weitmann ${ }^{\mathrm{b}}$ Andreas Greinacher ${ }^{\mathrm{a}}$ \\ Wolfgang Hoffmann ${ }^{b}$ \\ a Institut für Immunologie und Transfusionsmedizin, Universitätsmedizin Greifswald, Greifswald, Germany; \\ ${ }^{\mathrm{b}}$ Institut für Community Medicine, Universitätsmedizin Greifswald, Greifswald, Germany
}

\section{Keywords}

Red blood cell concentrates · Blood supply · Demographic change

\begin{abstract}
Background: Annual transfusion rates in many European countries range between 25 and 35 red blood cell concentrates $(\mathrm{RBCs}) / 1,000$ population. It is unclear why transfusion rates in Germany are considerably higher (approx. 50-55 $\mathrm{RBCs} / 1,000$ population). Methods: We assessed the characteristics of transfusion recipients at all hospitals of the German federal state Mecklenburg-Western Pomerania during a 10 -year longitudinal study. Results: Although $75 \%$ of patients received $\leq 4 \mathrm{RBC} /$ patient in 2015 (1 RBC: 11.3\%; 2 RBCs: 42.6\%; 3 RBCs: 6.3\%; 4 RBCs: 15.0\%), the mean transfusion index was 4.6 RBCs due to a minority of patients with a high transfusion demand. Two thirds of all RBCs were transfused to only $25 \%$ of RBC recipients. Consistently, male patients received a higher number of RBCs (2005: 54.2\%; 2015: $56.8 \%$ ) and had a higher mean transfusion index than female patients (mean 5.1 \pm 7.2; median 2; inter-quartile range [IQR] $2-4$ vs. mean $4.0 \pm 5.8$; median 2 ; IQR $2-4$ ). The absolute transfusion demand decreased between 2005 and 2015 by $13.5 \%$ due to a composite of active reduction (clinical practice change) and population decline in the 65- to 75-year age group (lower birth rate cohort 1940-1950); however, with major differences between hospitals (range from -61.0 to $+41.4 \%)$. Conclusion: Transfusion demand in a population
\end{abstract}

could largely be driven by patients with high transfusion demand. Different treatment practices in this group of patients probably add to the major differences in transfusion demand per 1,000 individuals between countries. The available data cannot prove this hypothesis. Implementation of a diagnosis-related group-based monitoring system is urgently needed to allow informative monitoring on the population level and meaningful comparisons between transfusion practices.

(C) 2020 The Author(s)

Published by S. Karger AG, Basel

\section{Introduction}

The impact of demographic changes on blood supply and transfusion demand has been addressed by a number of groups in Europe, North America, and Asia [1-17]. In many regions the numbers of blood donations are decreasing due to low birth rates, and numbers of patients increasing due to an increase in the older population. Already in regions in Germany $[1,5,10,18]$, Switzerland [8], the Netherlands [3], Finland [9], and also outside Europe in the USA [6], Canada [4], China [7], and Japan [2], a shortage in blood supply has been observed or forecast for the next few years. We performed a longitudinal study between 2005 and 2015 in the German federal state of Mecklenburg-Western Pomerania to analyze the impact of demographic changes on blood supply and demand on the population level. Data of all whole-blood donors and

(C) 2020 The Author(s)

Published by S. Karger AG, Basel

This is an Open Access article licensed under the Creative Common Attribution-NonCommercial-4.0 International License (CC BY-NC) (http://www.karger.com/Services/OpenAccessLicense), applicable to the online version of the article only. Usage and distribution for commercial purposes requires written permission.
Andreas Greinacher

Institut für Immunologie und Transfusionsmedizin Universität Greifswald

Sauerbruchstrasse, DE-17489 Greifswald (Germany)

andreas.greinacher@med.uni-greifswald.de 
all red blood cell concentrate (RBC) recipients of the entire federal state were collected $[1,5]$. In the federal system of Germany organization of health care is an obligation of each of the federal states. Our data were obtained from the health care system in Mecklenburg-Western Pomerania, a state in the north-east of Germany with 1.6 million inhabitants, who represented about $2.0 \%$ of the German population in 2015. Our study closely reflects the trends of health care changes on the population level, as only very few patients of the region are treated in other federal states, and vice versa.

In our previously published analyses of this study, we observed that male patients, who represented $49.6 \%$ of the population in 2005 and $49.5 \%$ in 2015, received $54.2 \%$ of RBCs in 2005 and $56.8 \%$ of RBCs in 2015 [1, 5]. This has been addressed by a more detailed sub-analysis in the present study.

We also noticed that most RBCs were transfused to patients older than 65 years and, although this age group increased by 3.3\% from 2005 to 2015 in MecklenburgWestern Pomerania, the total RBC demand declined by 7,568 RBCs $(-11.2 \%)[1,5]$. To better understand whether this trend is primarily the result of a reduced transfusion demand per 1,000 population in this age group, or whether also demographic factors are involved, we now assessed the correlation of the demographic changes in Mecklenburg-Western Pomerania with the development of RBC transfusion demand.

Like in other countries, the transfusion demand of patients in Mecklenburg-Western Pomerania decreased by $13.5 \%$ from 2005 to 2015 [1]. This decline in transfusion demand is usually attributed to the effects of improved patient blood management programs $[8,11,12,19]$. The transfusion rate of 51/1,000 population in 2015 in our study is close to the nationwide transfusion rate of $55 / 1,000$ population in Germany [20]. However, despite the recent decline, the transfusion rates in Germany are much higher than corresponding rates in other European countries, for example in the Netherlands (27/1,000 population) or Switzerland (35/1,000 population) [20]. The reasons for these major differences are currently unclear. Due to a lack of data, little information is available on the characteristics of the transfusion recipients in Germany [10]. Here we use the data from our prospective study to further analyse transfusion indices and characteristics of the recipients of RBCs in the state of Mecklenburg-Western Pomerania.

\section{Methods}

For each transfused RBC in Mecklenburg-Western Pomerania in the years 2005 and 2015 the following characteristics of the recipient were determined: age (or date of birth), sex, patient classification (surgical, medical, critically ill/emergency room, pediat- ric [according to the ward in which the patient was treated]), and date of transfusion. These data were provided by all 40 hospitals in the federal state $[1,5,18,21]$. Pediatric and non-classifiable patients were not analyzed in detail. These groups accounted for less than $3 \%$ of all RBC transfusions.

The hospitals were categorized by the number of beds in 2015 into small ( $<400$ beds), medium (400-700 beds), and large hospitals (>700 beds) [22]. In total 82,591 RBCs were transfused in 2015. Among these a definite assignment of the transfused RBC to an individual patient was possible for 54,665 RBCs based on pseudonymized patient identity numbers, corresponding to $66 \%$ of all transfused RBCs in 2015 (for the other patients no identifier was provided). Pseudonymized patient identity numbers could be provided for 41,963 patients of large hospitals (76.5\% of this sub-analysis), for 3,822 patients of medium-sized hospitals (7.0\%), and for 9,062 patients of small hospitals (16.5\%). Based on this data set the transfusion index was determined for different hospital and patient categories. The transfusion index is defined as the number of RBCs transfused per transfused patient. We used the SAS statistical software package (v9.4; SAS Institute Inc., Cary, NC, USA) to calculate descriptive statistics. Categorical data are expressed as percentages; continuous data are expressed as the mean ( \pm 2 standard deviations; SD) or median (and interquartile range; IQR). Differences between groups were tested using the $\chi^{2}$ test. A $p$ value $<0.05$ was considered statistically significant.

Population data in 1-year age categories for 2005 and 2015 were obtained by the population registry for Mecklenburg-Western Pomerania [23-25]. These data were used to calculate gender- and age-specific transfusion rates per 1,000 inhabitants.

\section{Results}

\section{RBC Demand by Patient Characteristics}

Based on a sub-analysis of 54,665 RBCs transfused to 12,011 patients (with available pseudonymized patient identity numbers), which corresponded to $66 \%$ of all transfused RBCs in Mecklenburg-Western Pomerania in 2015, the transfusion index was determined. Most patients received 2 RBCs per patient (42.6\%), followed by 4 RBCs per patient (15.0\%) and 1 RBC per patient (11.3\%; Fig. 1). Overall, $75 \%$ of all patients were transfused with fewer than 5 RBCs. However, the mean transfusion index was 4.6 RBCs ( \pm 6.6 ; median 2; IQR 2-4) per patient, due to a small number of patients with an exceedingly high demand (up to 202 RBCs per patient within 1 year). This is underlined by the fact that only $25 \%$ of all patients who were transfused with more than 4 RBCs received about $66 \%$ of all RBCs.

The transfusion indices differed depending on sex, classification, and hospital size (Table 1). Male patients had a higher mean transfusion index than female patients (5.1 \pm 7.2 vs. $4.0 \pm 5.8$ per patient; median 3 [IQR 2-4] vs. 2 [IQR 2-4] RBCs per patient). In all patient categories the mean RBC demand per patient was highest in large hospitals, while the median transfusion index did not differ between hospitals. Critically ill and emergency patients had the highest mean transfusion indices irrespective of hospital size. For these patients also the median 
Fig. 1. Absolute number of patients and transfused RBCs for different transfusion indices $(n=12,011$ patients transfused with 54,665 RBCs). Most patients received 4 or fewer RBCs in 2015. However, the majority of RBCs were transfused to only those $25 \%$ of all patients who received more than 4 RBCs per patient.

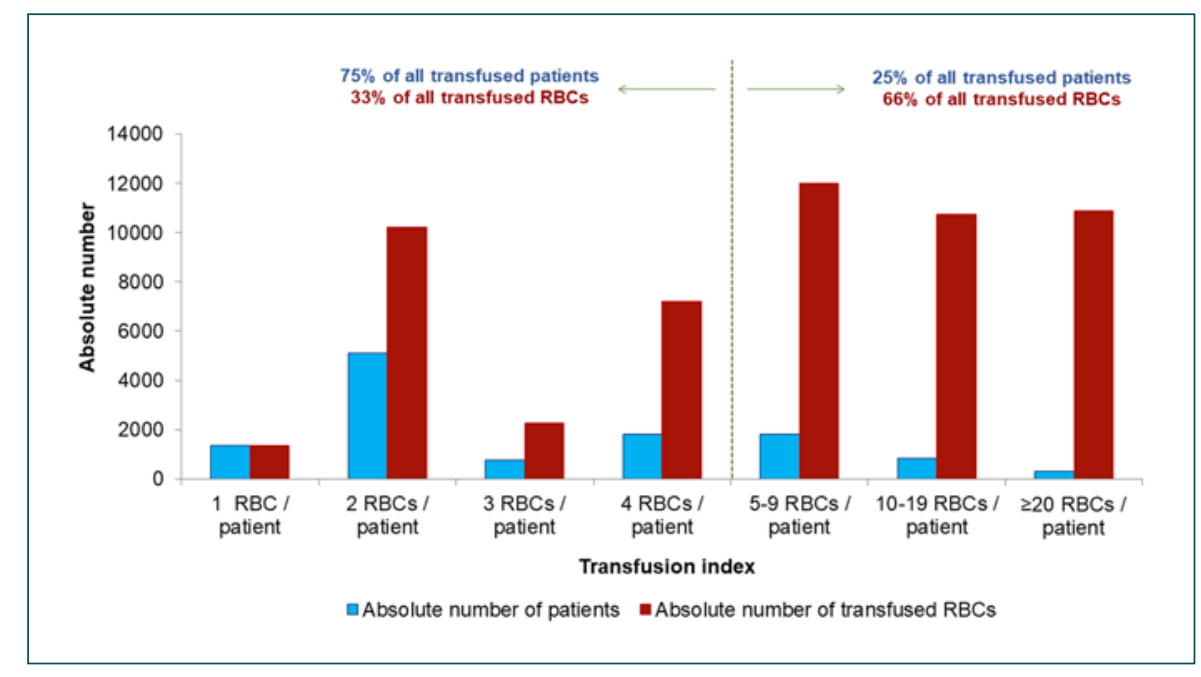

Table 1. Mean and median transfusion indices by sex, hospital size, and patient categories

Mean transfusion index $(\mathrm{SD})^{1}$; median transfusion index $(\mathrm{IQR})$

\begin{tabular}{ll}
\hline Overall $^{2}$ & $4.6(6.6) ; 2(2-4)$ \\
Males & $5.1(7.2) ; 3(2-6)$ \\
Females & $4.0(5.8) ;(2-4)$
\end{tabular}

\begin{tabular}{llll}
\hline & Surgical & Critically ill & Medical \\
\hline Males & $3.7(3.7) ; 2(2-4)$ & $5.7(8.9) ; 3(2-6)$ & $4.1(5.8) ; 2(2-4)$ \\
Females & $3.1(2.9) ; 2(2-4)$ & $4.7(8.3) ; 2(2-4)$ & $3.4(4.5) ; 2(2-4)$ \\
Small hospitals (<400 beds) & $3.2(3.0) ; 2(2-4)$ & $4.5(4.9) ; 2(2-6)$ & $2.9(2.5) ; 2(2-4)$ \\
Medium hospitals (400-700 beds) & $2.6(1.3) ; 2(2-2)$ & $4.3(5.2) ; 2(2-5)$ & $3.0(2.5) ; 2(2-4)$ \\
Large hospitals (>700 beds) & $3.5(3.5) ; 2(2-4)$ & $5.7(10.0) ; 3(2-6)$ & $4.2(6.0) ; 2(2-4)$ \\
\hline
\end{tabular}

${ }^{1}$ The mean transfusion index is defined as the number of RBCs transfused per transfused patient in the respective subgroup.

${ }^{2} n=12,011$ patients transfused with 54,665 RBCs.

transfusion index was higher in large hospitals compared to medium and small hospitals (3 vs. 2 RBCs/patient). All these effects are caused by higher numbers of patients with an exceedingly high RBC demand in the respective groups (online suppl. Table 1; for all online suppl. material, see www.karger.com/doi/10.1159/000510207).

The transfusion demand for RBCs declined in all patient groups between 2005 and 2015. This reduction was less pronounced in males than in females in both surgical ( $-9.2 \mathrm{vs}$. $-25.8 \% ; p<0.01)$ and in medical patients ( -6.8 vs. $-13.4 \%$; $p<0.01$ ), while in critically ill patients the decrease was similar in males and females (-7.8 vs. $-7.2 \%$; $p=0.73)$.

\section{RBC Demand by Hospital Characteristics}

We analyzed 5 large hospitals with more than 700 beds, 3 medium-sized hospitals (400-700 beds), and 21 small hospitals with fewer than 400 beds. In 2015, 56.1\% of all RBCs were transfused in large hospitals $(46,303$ RBCs), $13.8 \%$ in medium-sized hospitals (11,393 RBCs), and $30.1 \%$ in small hospitals (24,895 RBCs; Fig. 2).
The different hospital categories showed different age distributions of patients receiving RBCs (Fig. 3). Independent of hospital size, patients in the age group 70-84 years had the highest RBC demand of all age groups (small: $45.2 \%$, medium: $47.5 \%$, large: $42.8 \%$ ). Small hospitals transfused more RBCs to patients in the age group 45-54 years (17.3\%) compared to medium (7.5\%) and large hospitals (10.9\%). This trend was observed in surgical and medical patients, but not in critically ill patients. The proportion of transfused RBCs to patients in the age group $\geq 75$ years was higher in medium-sized hospitals (47.1\%) than in small (45.5\%) or large hospitals (37.0\%).

The reduction of the transfusion demand between 2005 and 2015 differed among hospital categories (Fig. 2). In small hospitals $17.7 \%$ fewer $(-5,351)$, in medium-sized hospitals $18.6 \%$ fewer $(-2,595)$, and in large hospitals $9.6 \%$ fewer $(-4,918)$ RBCs were transfused. However, the heterogeneity between hospitals regarding the development of RBC demand between 2005 and 2015 was remarkable (with a range of -61.0 to $+41.4 \%$ in small hos- 


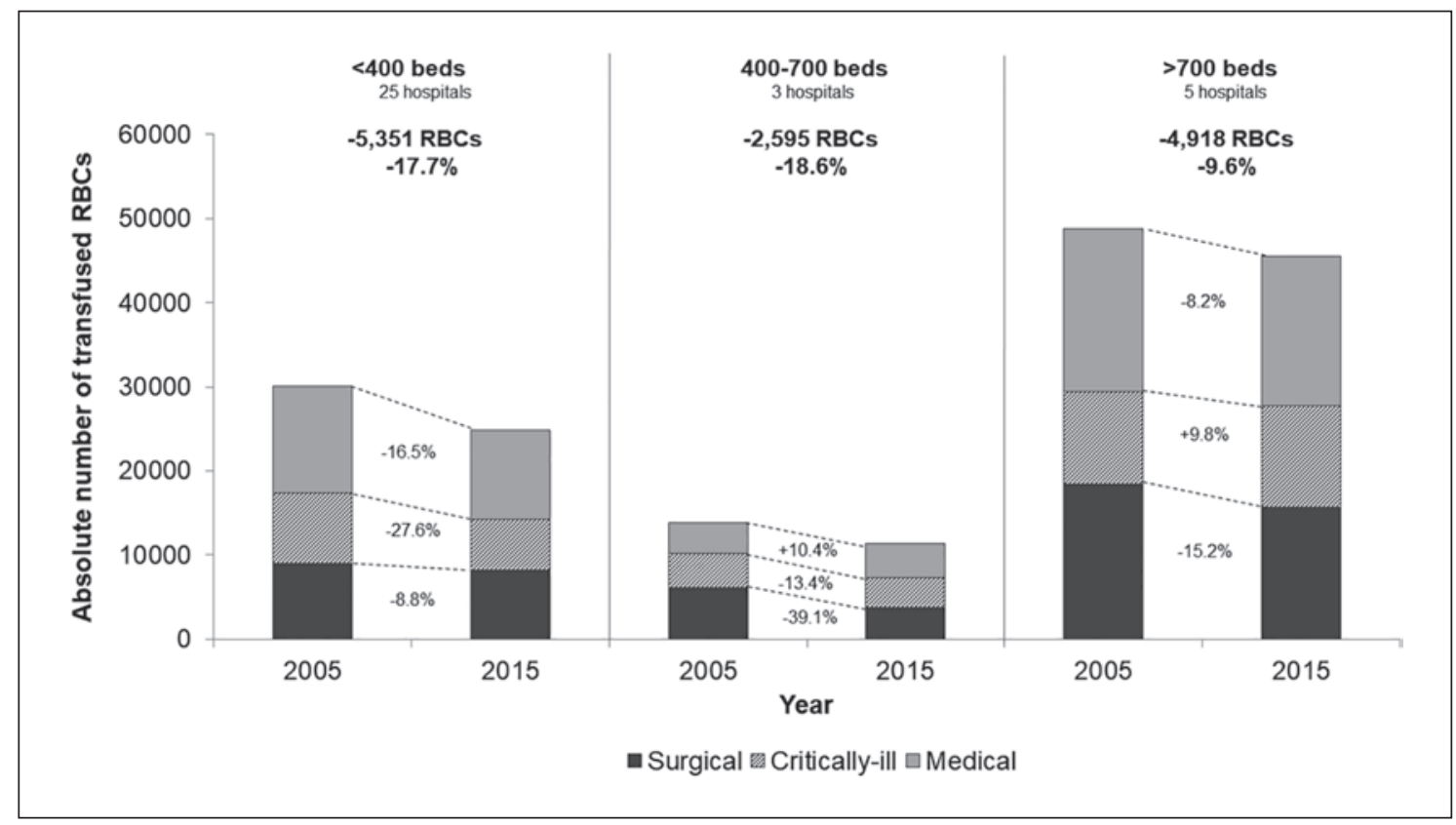

Fig. 2. Absolute number of transfused RBCs in hospitals of different sizes in 2005 and 2015. All hospitals showed a decrease in transfusion demand between 2005 and 2015. This was more pronounced in medium and smaller hospitals compared to large hospitals. While the transfusion demand for surgical patients decreased in all hospital categories, medical patients required more RBCs in medium-sized and critically ill patients required more RBCs in large hospitals, respectively.

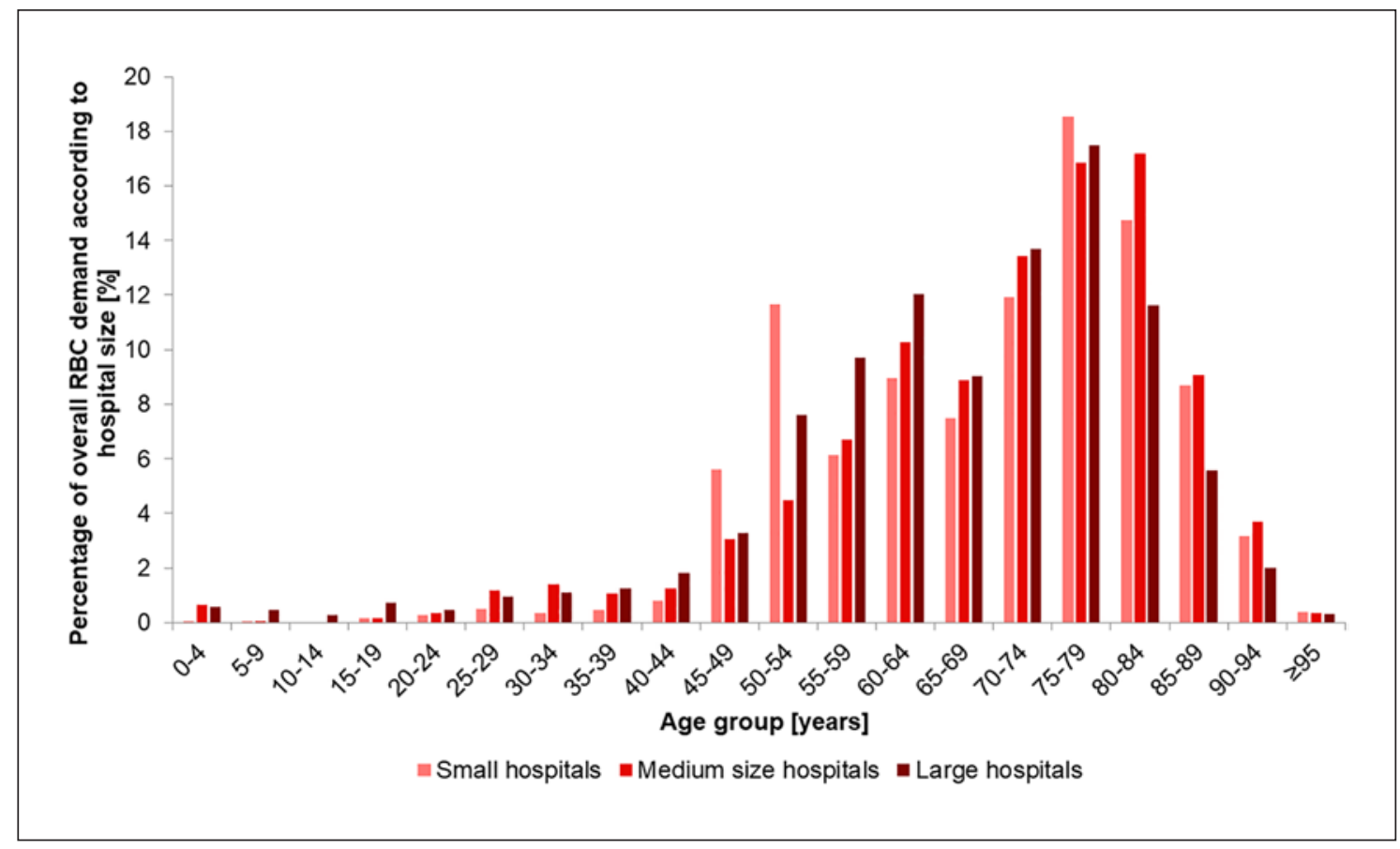

Fig. 3. Proportion of overall RBC demand per age group according to hospital size. In all hospitals most RBCs were transfused to patients aged 70-84 years. Otherwise, the number of transfused RBCs per age group differs depending on hospital size. The proportion of RBCs transfused to patients aged 50-69 years is highest in large hospitals, whereas the proportion of RBCs transfused to patients $\geq 80$ years is higher in small and medium-sized hospitals. However, there is a relatively high proportion of RBCs transfused to patients aged 45-54 years in small hospitals, which was not observed in medium and large hospitals. 

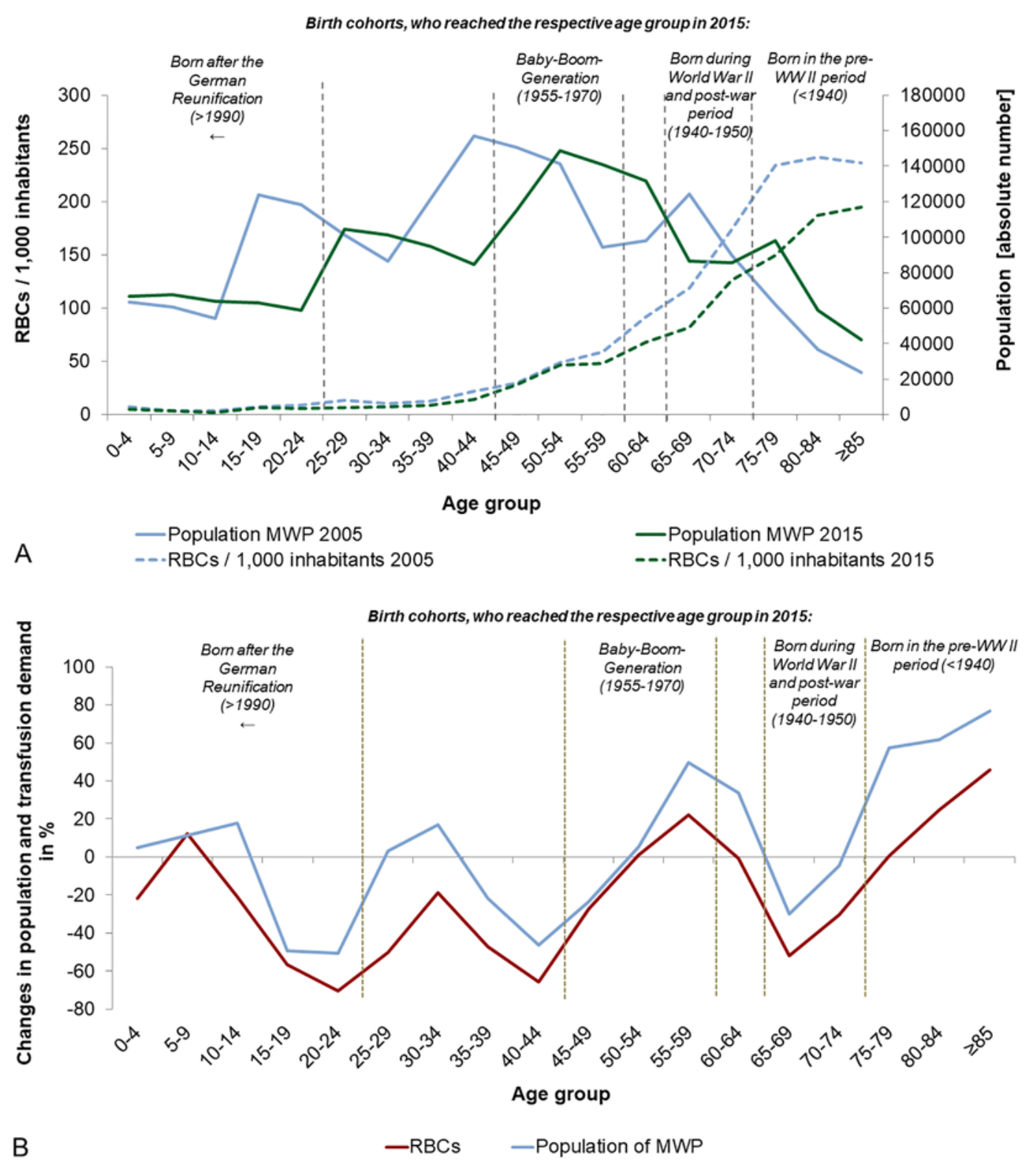

Fig. 4. A Absolute number of inhabitants in Mecklenburg-Western Pomerania (MWP; solid lines) and the respective number of transfused RBCs per 1,000 inhabitants per age group (dotted lines) in 2005 (blue) and 2015 (green). In 2015 the low birth rate cohort of World War II and the post-war period (65-74-year age group) contributed to the decrease in absolute transfusion demand due to a reduced absolute number of inhabitants. This has a substantial effect on the total transfusion demand, as these age groups have high transfusion rates. In the near future the baby-boom generation (4559 years old in 2015) will shift towards the age groups with the highest transfusion rates, which will likely cause an increase in transfu-

pitals, -24.9 to $-11.8 \%$ in medium hospitals, and -23.7 to $+13.2 \%$ in large hospitals). The reduction of transfusion demand also depended on patient characteristics. Fewer RBCs were transfused in all hospital size categories in sur- sion demand. B Changes in the population numbers of Mecklenburg-Western Pomerania (MWP) and the transfusion demand from 2005 to 2015 per age group. The change in RBC demand per age group (red line) between the years 2005 and 2015 was always below the change in population numbers within the different age groups (blue line). This reflects the real decrease in transfusion demand due to changes in medical practice. However, the pronounced decrease in population numbers in the 65- to 75-year age group (World War II birth decline) and to a lesser extend in the 35- to 50year age group (low birth rate due to introduction of hormonal contraceptives) reduce the overall transfusion demand.

gical patients, whereas an increase of transfused RBCs was observed for medical patients in medium-sized hospitals $(+10.4 \%)$ and for critically ill patients in large hospitals (+9.8\%; Fig. 2). 
Reduction of the Transfusion Demand Is a Composite of Active Reduction and Demographic Changes

In 2005 and 2015 most RBCs were transfused to patients older than 65 years (Fig. 4A, hatched lines). An important reason for the trend of decreasing $\mathrm{RBC}$ demand in this age group was the population decline of the 65- to 75 -year age group due the low birth rate cohort of World War II and the post-war period born between 1940 and 1950 (online suppl. Table 2).

As shown in Figure 4B, the development of RBC demand from 2005 to 2015 followed the increase and decrease of the population size of each age group, i.e., whenever the population of one age group increased, the RBC demand increased, and vice versa.

However, the reduction of RBC demand (red line in Fig. 4B) was always more pronounced than the reduction in population numbers (except the 5- to 9-year age group; blue line in Fig. 4B) and the increase of transfusion demand was not as pronounced as the increase of the respective population group. This difference between transfusion demand and changes in the population numbers of $25.5 \%$ reflects the true effects of changes in transfusion practice. This is also reflected in Figure $4 \mathrm{~A}$ where the green line (population in 2015) is above the blue line (population in 2005) in the 55- to 65-year and 75- to $>85$ year age groups, but still the transfusion demand per thousand inhabitants in these age groups was lower than in 2005 (the hatched green line is below the hatched blue line).

\section{Discussion}

This study presents the characteristics of patients receiving RBC transfusions in the German federal state Mecklenburg-Western Pomerania. The decrease in transfusion demand between 2005 and 2015 by $-13.5 \%$ is similar to observations in other countries. An absolute reduction of RBC transfusions of $-9.5 \%$ was observed in Switzerland between 2003 and 2013 [8] and of $-20 \%$ in the Netherlands between 1996 and 2005 [12]. In Austria, a relative reduction in transfusion rates of $-27 \%$ was observed in selected orthopedic procedures from 2005 to 2010, whereas transfusion rates in cardiac surgery remained relatively constant [19]. Also, in northern England a reduction in transfusion rates of $-21 \%$ was observed $[11,26]$. Changes in transfusion practice are usually thought to be the main cause of this trend. However, the population decline due to the low birth rate cohort during World War II and the post-war period is another important reason. A similar observation was made by Brockmann et al. [27] (presented at the DGTI congress, Lübeck, 2018), who compared demographic changes with the development of RBC transfusions and observed a decrease of RBC demand due to the decline of the birth cohort during World War II. This is an important observation. Unless the transfusion rate per 1,000 population in the age group $>65$ years further declines (due to changes in medical practice), the trend of a decrease in absolute transfusion demand will inverse, when the baby-boom generation reaches the age groups with the highest transfusion rates. In this regard Central Europe differs considerably from North America, where the proportion of younger age groups is much higher.

Not surprisingly, we found higher mean transfusion indices in critically ill patients than in medical or surgical patients and the highest transfusion indices in large hospitals, whereas the median transfusion indices did not differ substantially between hospital categories. This can be explained by the fact that the mean transfusion indices are largely driven by a few patients with an extremely high demand. This also explains why the transfusion demand in our study is primarily driven by patients requiring more than 4 RBCs per year, with $25 \%$ of transfused patients receiving $66 \%$ of all RBCs. This is probably a more general feature, as the PROTON study performed in the Netherlands also reported that more than $65 \%$ of all RBCs were transfused to patients with transfusion indices of $>4$ RBCs within 1 year [28].

Transfusion rates differ remarkably between countries [20], and differences in medical practice in situations which require maximal therapy could be part of a possible explanation. Differentiation between patients with transfusion indices of $\leq 4$ or $>4$ RBCs per patient should be considered in regional and especially international comparisons of transfusion demands, and both parameters, along with the mean and the median transfusion index, should be used for comparison.

Typically, patients with a high transfusion demand are severely sick. This is a likely explanation for the less pronounced reduction of RBC demand we observed in large hospitals. Attention should be paid to this fact in future studies on changes in the transfusion demand. If only large hospitals are enrolled into a study, the results might not reflect the real development in RBC transfusion requirements. This is further underscored by our finding that hospitals of different sizes show a different age distribution of transfused patients. This finding is again consistent with the results of the PROTON study in the Netherlands [28]. As patients in large hospitals are usually more likely to participate in clinical studies and larger hospitals are often better equipped with an electronic hospital information system [5], hospital size is likely a relevant source of selection bias. In addition, any study on the development of transfusion demand over time should include a large enough number of hospitals, as we observed remarkable differences in the changes in transfusion demand between individual hospitals. These differ- 
ences are probably not only caused by different transfusion strategies. Instead, structural changes, for example the implementation of new procedures or establishment of new departments (like hemato-oncology), are important underlying reasons. As we have no data on the exact underlying disease groups it is difficult to further interpret these numbers. For example, the higher transfusion numbers in patients $>75$ years in small and medium-sized hospitals might be due to a more liberal transfusion policy or due to more palliative care patients being treated in these hospitals. A major strength of our study is that we included all hospitals of the state, which avoids selection bias in the analyses. Changes in patient characteristics of an individual hospital level out because an increase of one patient group in one hospital will always be accompanied by a decrease of the same patient group in other hospitals, as health care is predominantly maintained within the state and "patient tourism" to other states or vice versa is rare.

Finally, we observed that both the absolute transfusion demand as well as the transfusion indices were consistently higher in male patients compared to female patients. Such gender-related differences in the transfusion demand have also been observed by others $[3,8,11,16$, $28-31]$. However, there is no explanation for this in the literature. This may reflect the difference in blood volume and body mass between males and females, or a higher rate of cardiovascular diseases and cardiac surgeries in males, or a different approach of physicians to male and female patients. This interesting observation should be further studied.

Because we could not include the specific diagnoses of the transfused patients, we cannot further interpret our observation. Implementation of the standardized Diagnosis-Related-Group (DRG) system and a DRG-based monitoring system would allow a better comparison between transfusion strategies of different hospitals and much more detailed analyses.

Our study is the largest longitudinal study on transfusion demand and blood donation numbers in a defined geographical region. The interactions of different pa- rameters influencing the transfusion demand are much more complex than we had anticipated at the onset of our study. In times of major demographic changes associated with an increased risk of blood shortages and major changes in clinical practice in surgical as well as hemato-oncological patients, it is urgent to implement a nationwide, DRG-based monitoring system of RBC transfusions across all age groups of the population to obtain realistic data for planning of the future blood supply.

\section{Acknowledgements}

We thank all hospitals in Mecklenburg-Western Pomerania for providing their data on blood transfusions.

\section{Statement of Ethics}

Exclusively anonymous data were used in this study. The institutional ethics review board of the University Medicine Greifswald approved the study. All authors had full access to all data including all statistical reports and tables used in the manuscript.

\section{Conflict of Interest Statement}

All authors declare that they have no conflicts of interest.

\section{Funding Sources}

No external funding was received.

\section{Author Contributions}

W.H. and A.G. conceived and designed the study. K.W., L.S., W.H., and A.G. acquired, analyzed, or interpreted the data, drafted the article or provided critical revision for important intellectual content, and provided final approval of the version to be published. All authors are accountable for all aspects of the work in ensuring that questions related to the accuracy or integrity of any part of the article are appropriately investigated and resolved.

\section{References}

1 Greinacher A, Weitmann K, Schönborn L, Alpen U, Gloger D, Stangenberg W, et al. A population-based longitudinal study on the implication of demographic changes on blood donation and transfusion demand. Blood Adv. 2017 May;1(14):867-74.

2 Akita T, Tanaka J, Ohisa M, Sugiyama A, Nishida K, Inoue S, et al. Predicting future blood supply and demand in Japan with a Markov model: application to the sex- and age-specific probability of blood donation. Transfusion. 2016 Nov;56(11):2750-9.
3 Borkent-Raven BA, Janssen MP, Van Der Poel CL. Demographic changes and predicting blood supply and demand in the Netherlands. Transfusion. 2010 Nov;50(11):2455-60.

4 Drackley A, Newbold KB, Paez A, Heddle N. Forecasting Ontario's blood supply and demand. Transfusion. 2012 Feb;52(2):366-74.

5 Schönborn L, Weitmann K, Greger N, Kiefel V, Hoffmann W, Greinacher A. Longitudinal changes in the blood supply and demand in North-East-Germany 2005-2015. Transfus Med Hemother. 2017 Aug;44(4):224-31.
6 Sullivan MT, Cotten R, Read EJ, Wallace EL. Blood collection and transfusion in the United States in 2001. Transfusion. 2007 Mar; 47(3):385-94

7 Shi L, Wang JX, Stevens L, Ness P, Shan H. Blood safety and availability: continuing challenges in China's blood banking system. Transfusion. 2014 Feb;54(2):471-82.

8 Volken T, Buser A, Castelli D, Fontana S, Frey BM, Rusges-Wolter I, et al. Red blood cell use in Switzerland: trends and demographic challenges. Blood Transfus. 2018;16(1):73-82. 
9 Ali A, Auvinen MK, Rautonen J. The aging population poses a global challenge for blood services. Transfusion. 2010 Mar;50(3):584-8.

10 Seifried E, Klueter H, Weidmann C, Staudenmaier T, Schrezenmeier H, Henschler R, et al. How much blood is needed? Vox Sang. 2011 Jan;100(1):10-21.

11 Tinegate H, Pendry K, Murphy M, Babra P, Grant-Casey J, Hopkinson C, et al. Where do all the red blood cells (RBCs) go? Results of a survey of RBC use in England and North Wales in 2014. Transfusion. 2016 Jan;56(1) 139-45.

12 van Hoeven L, Koopman R, Koffijberg H, Roes K, Janssen M. Historical time trends in red blood cell usage in the Netherlands. IJCTM. 2016;4:67-77.

13 Jóhannsdóttir V, Gudmundsson S, Möller E, Aspelund T, Zoëga H. Blood donors in Iceland: a nationwide population-based study from 2005 to 2013. Transfusion. 2016 Jun; 56(6 Pt 2):1654-61

14 Lattimore S, Wickenden C, Brailsford SR. Blood donors in England and North Wales: demography and patterns of donation. Transfusion. 2015 Jan;55(1):91-9.

15 Barr PJ, Donnelly M, Morris K, Parker M, Cardwell C, Bailie KE. The epidemiology of red cell transfusion. Vox Sang. 2010 Oct; 99(3):239-50

16 Madsen JT, Kimper-Karl ML, Sprogøe U, Georgsen J, Titlestad K. One-year period prevalence of blood transfusion. Transfus Med. 2010 Jun;20(3):191-5.

17 García-Erce JA, Campos A, Muñoz M. Blood donation and blood transfusion in Spain (1997 - 2007): the effects of demographic changes and universal leucoreduction. Blood Transfus. 2010 Apr;8(2):100-6.

18 Greinacher A, Weitmann K, Lebsa A, Alpen U, Gloger D, Stangenberg W, et al. A population-based longitudinal study on the implications of demographics on future blood supply. Transfusion. 2016 Dec;56(12):2986-94.
19 Gombotz H, Rehak PH, Shander A, Hofmann A. The second Austrian benchmark study for blood use in elective surgery: results and practice change. Transfusion. 2014 Oct;54(10 Pt 2):2646-57.

20 van Hoeven LR, Janssen MP, Rautmann G: The collection, testing and use of blood and blood components in Europe: European Committee (Partial Agreement) on Blood Transfusion. EDQM Report, 2013. pp. 1-48.

21 Greinacher A, Fendrich K, Brzenska R, Kiefel V, Hoffmann W. Implications of demographics on future blood supply: a populationbased cross-sectional study. Transfusion. 2011 Apr;51(4):702-9.

22 Hunz M. Krankenhausplan 2012: des Landes Mecklenburg-Vorpommern, Land Mecklenburg-Vorpommern. 2012. https://www. regierung-mv.de/Landesregierung/wm/gesundheit/Gesundheitsversorgung/Krankenhauswesen/ (accessed June 28, 2018).

23 Statistisches Amt Mecklenburg-Vorpommern. A1832 Aktualisierte 4. Landesprognose (Basisjahr 2010): Bevölkerungsentwicklung des Landes sowie der kreisfreien Städte und Landkreise bis 2030 nach Einzelalter. Schwerin, Statistisches Amt Mecklenburg-Vorpommern, 2013. https://www.destatis.de/GPStatistik/servlets/.../MVHeft.../A1832_2012_01.pdf (accessed March 15, 2017).

24 Statistisches Bundesamt. Bevölkerung. Bundesländer, Stichtag, Geschlecht, Altersjahre. Wiesbaden, Statistisches Bundesamt. 2017. https://www-genesis.destatis.de/genesis/online $/$ data;jsessionid $=54$ FFE50E1230826B019 6D310F6DD646D.tomcat_GO_1_1?operatio $\mathrm{n}=$ abruftabelleBearbeiten \&levelindex $=2 \&$ lev elid $=1486816342782$ \&auswahloperation $=a b$ ruftabelleAuspraegungAuswaehlen\&auswahl verzeichnis $=$ ordnungsstruktur\&auswahlziel=werteabruf\&selection name $=12411$ 0012\&auswahltext $=\% 23$ SDLAND-13\%23Z31.12.2015\%2C31.12.2010\%2C31.12.2005\& werteabruf $=$ Werteabruf (accessed February 11, 2017).
25 Statistisches Bundesamt. Bevölkerung der Bundesländer nach Einzeljahren. Wiesbaden, Statistisches Bundesamt, Wiesbaden. 2017. https://www-genesis.destatis.de/genesis/online/data;jessionid=5E65E2424BDC01A2E5 4B574C54C336F1.tomcat GO 1 1 1 operatio $\mathrm{n}=$ ergebnistabelleUmfang\&levelindex $=3 \&$ le velid $=1486815164546 \&$ downloadname $=124$ 11-0011 (accessed February 11, 2017).

26 Tinegate $\mathrm{H}$, Chattree S, Iqbal A, Plews D, Whitehead J, Wallis JP; Northern Regional Transfusion Committee. Ten-year pattern of red blood cell use in the North of England. Transfusion. 2013 Mar;53(3):483-9.

27 Brockmann C, Hagen C, Görg S. Demography in Germany is a cause of the present decrease in the number of transfused red blood cell concentrates (RBC) and possibly of a future increase. 51. Jahrestagung der Deutschen Gesellschaft für Transfusionsmedizin und Immunhämatologie (DGTI), Lübeck, September 19-21, 2018 [Abstract]. Transfus Med Hemother. 2018;45:35.

28 Borkent-Raven BA, Janssen MP, van der Poel CL, Schaasberg WP, Bonsel GJ, van Hout BA. The PROTON study: profiles of blood product transfusion recipients in the Netherlands. Vox Sang. 2010 Jul;99(1):54-64.

29 Bruun MT, Pendry K, Georgsen J, Manzini P, Lorenzi M, Wikman A, et al. Patient Blood Management in Europe: surveys on top indications for red blood cell use and Patient Blood Management organization and activities in seven European university hospitals. Vox Sang. 2016 Nov;111(4):391-8.

30 Kamper-Jørgensen M, Edgren G, Rostgaard K, Biggar RJ, Nyrén O, Reilly M, et al. Blood transfusion exposure in Denmark and Sweden. Transfusion. 2009 May;49(5):888-94.

31 Valero-Elizondo J, Spolverato G, Kim Y, Wagner D, Ejaz A, Frank SM, et al. Sex- and age-based variation in transfusion practices among patients undergoing major surgery. Surgery. 2015 Nov;158(5):1372-81. 
